\title{
Neoadjuvant radiation therapy for esophageal cancer: is a higher dose better?
}

\author{
Waqar Haque, E. Brian Butler, Bin S. Teh \\ Department of Radiation Oncology, Houston Methodist Hospital, Houston, TX, USA \\ Correspondence to: Waqar Haque, MD. Department of Radiation Oncology, Houston Methodist Hospital, Cancer Center and Research Institute, Weil \\ Cornell Medical College, Houston, TX 77030, USA. Email: waqarh786@gmail.com. \\ Provenance: This is an invited article commissioned by the Academic Editor Dr. Shuangjiang Li (Department of Thoracic Surgery and West China \\ Medical Center, West China Hospital, Sichuan University, Chengdu, China). \\ Comment on: Semenkovich TR, Samson PP, Hudson JL, et al. Induction Radiation Therapy for Esophageal Cancer: Does Dose Affect Outcomes? \\ Ann Thorac Surg 2019;107:903-11.
}

Submitted Nov 15, 2019. Accepted for publication Nov 21, 2019.

doi: $10.21037 /$ jtd.2019.11.77

View this article at: http://dx.doi.org/10.21037/jtd.2019.11.77

Neoadjuvant chemoradiation (CRT) is currently a standard of care for locally advanced esophageal cancer (1). The data supporting the efficacy of neoadjuvant CRT over surgery alone is supported by multiple randomized trials, the largest of which was the CROSS trial, which showed that for patients with locally advanced esophageal cancer, treatment with 41.4 Gy in 23 fractions with concurrent Carboplatin/Taxol results in improved overall survival (OS) when compared to treatment with surgery alone (2). The CALGB 9781 trial randomized patients to neoadjuvant treatment with 50.4 Gy in 28 fractions with concurrent cisplatin and fluouracil or surgery alone, and demonstrated superior OS in the group of patients receiving neoadjuvant treatment, but this trial closed early due to poor accrual (3). Other randomized trials have used neoadjuvant radiation therapy (RT) doses between 35-50 Gy along with chemotherapy with varying results (4-6). Therefore, the optimal neoadjuvant RT dose is a matter of debate, and the current NCCN Guidelines recommend a radiation dose of 41.4-50.4 Gy.

Now that neoadjuvant CRT has been established as a standard of care for patients with locally advanced esophageal cancer, it is important to determine the optimal neoadjuvant RT dose since this can have meaningful clinical impact into the outcomes of patients. A higher neoadjuvant RT dose has been shown to increase the change of a surgical resection with negative margins and in doing so prolong OS $(2,6)$. That being said, dose escalation should be done with caution, as higher neoadjuvant RT doses have also been associated with poor healing of the esophagogastric anastomosis and postoperative morbidity (7). Dose-escalation studies for esophageal cancer have not been performed in the neoadjuvant setting. Studies that have compared 64.8 to 50.4 Gy as definitive treatment for esophageal cancer has demonstrated no benefit to dose escalation $(8,9)$.

It is in this context that Semenkovich et al. (10) conducted a large retrospective study using a contemporary national dataset to provide further clarity regarding the tumor response, perioperative mortality, and OS differences for patients with locally advanced esophageal cancer receiving neoadjuvant RT followed by esophagectomy, when stratified by neoadjuvant RT dose. Patient records were obtained from the National Cancer Data Base $(\mathrm{NCDB})$, and inclusion criteria included patients with either squamous carcinoma or adenocarcinoma of the esophagus diagnosed from 2004 to 2014 and treated with induction RT and esophagectomy. Patients were included if initiation of induction therapy and surgery were $<6$ months apart, and the radiation dose was 30-70 Gy. Patients were divided into three cohorts based on the neoadjuvant RT dose: low dose RT (IRT) patients received $<40$ Gy, standard dose RT (sRT) patients received 40 to 50.4 Gy, and high dose RT (hRT) received >50. 4 Gy. The primary outcomes that were assessed were OS, 90-day postoperative mortality, and pathologic complete response (pCR) rate. Univariable 
and multivariable analyses were performed to identify factors and outcomes associated with the three radiation dosage groups, and Kaplan Meier curves were performed to compare OS.

A total of 12, 675 patients met the selection criteria and were included in the study. Of these, $10.5 \%(\mathrm{n}=1,329)$ received $1 \mathrm{RT}, 84.7 \%(10,738)$ received sRT, and $4.8 \%$ $(\mathrm{n}=608)$ received hRT. Amongst patients treated with photon based treatment, $58 \%$ received treatment with intensity modulated radiation therapy (IMRT), while $42 \%$ received treatment with $3 \mathrm{D}$ treatment. Neoadjuvant chemotherapy was administered to $98.3 \%$ of patients, and chemotherapy was administered concurrently to $85.3 \%$ of patients. Amongst all patients, pCR rates were found to increase with increasing neoadjuvant radiation dose, with $11.7 \%, 16.2 \%$, and $21.0 \%(\mathrm{P}<0.001)$ of patients achieving pCR in the IRT, sRT, and hRT dose groups, respectively. Higher neoadjuvant RT doses were also associated with a higher rate of down pathologic downstaging $(52.0 \%$ in IRT, $56.4 \%$ for sRT, and $63.1 \%$ for hRT patients, $\mathrm{P}=0.001)$. Importantly, no significant differences in surgical margin status were observed when comparing the different neoadjuvant RT dose cohorts. In regression analysis, use of IRT was associated with a lower likelihood of pCR when compared to standard dose [odds ratio (OR), 0.67; $\mathrm{P}<0.001$ ] as well as a lower likelihood of pathologic downstaging $(\mathrm{OR}$, $0.85, \mathrm{P}=0.04)$ when compared to the use of sRT, but no statistically significant differences were observed between either pCR rate or pathologic downstaging when patients treated with sRT were compared to those treated with hRT. Comparisons of postoperative mortality demonstrated that 90 -day postoperative mortality was significantly increased in the hRT group (hRT $12.7 \%$, sRT $7.9 \%$, and IRT $7.9 \%$; $\mathrm{P}<0.001$ ), and on logistic regression, hRT was associated with increased 90 - day postoperative mortality when compared to sRT (OR, 1.59, 95\% CI, 1.21-2.09). No differences were observed in 90-day postoperative mortality when comparing patients treated with IRT to those treated with sRT. No differences were observed in long term OS between the three radiation dose cohorts.

The investigators also performed subgroup analyses between patients treated with a neoadjuvant RT dose of 45 Gy to those treated with 50.4 Gy, and amongst patients with either squamous cell carcinoma or adenocarcinoma histology. Patients treated with a neoadjuvant RT dose of 50.4 Gy were found to have higher pCR rates than those treated to $45 \mathrm{~Gy}(17.3 \%$ vs. $15.0 \%, \mathrm{P}=0.003)$, though there was no observed difference in either OS or 90-day postoperative mortality between the two dose groups. Amongst patients with esophageal squamous cell carcinoma, higher pCR rates were observed with increased neoadjuvant RT dose: $18.1 \%$ for $1 \mathrm{RT}, 26.3 \%$ for sRT, and $33.9 \%$ for hRT, $\mathrm{P}=0.003$. Importantly, worse 90 - day postoperative mortality was observed for patients in the hRT groups $(19.4 \%$ in the hRT cohort, $9.9 \%$ in the sRT cohort, and $11.8 \%$ in the $1 \mathrm{RT}$ cohort, $\mathrm{P}=0.0003$ ). Also, improved median OS was observed for patients treated with neoadjuvant sRT (42.6 vs. 34.8 months for hRT and 30.7 months for 1RT, $\mathrm{P}=0.03$ ). In the adenocarcinoma histology cohort, higher pCR rates were again observed with increased neoadjuvant RT dose $(10.0 \%$ for $1 \mathrm{RT}, 14.0 \%$ for sRT, and $16.8 \%$ for $h R T, P<0.001$ ), though no statistically significant differences were found when comparing either median OS or 90-day post operative mortality between the different neoadjuvant RT dose groups.

The investigators concluded that while hRT could result in greater $\mathrm{pCR}$ rates, this was also associated with greater 90- day post operative mortality and no median OS benefit. Therefore, the authors suggested that a neoadjuvant RT dose between 40-50.4 Gy could maximize the tumor response without increasing the perioperative morbidity, and that this should be the standard neoadjuvant RT dose.

This study had several strengths that make its results generalizable to the general US population. One, since this study used the NCDB as the dataset, it was able to use cancer statistics from about $70 \%$ of the US population, and as such, base its conclusions on a large number of patients. Two, the authors were meticulous about stratifying patients by neoadjuvant RT dose. The three dose cohorts were appropriately selected and this allowed the investigators to observe statistically significant differences in rates of both pCR and postoperative morbidity. Finally, the authors used postoperative mortality as a surrogate for morbidity during surgery, and in doing so were able to account for treatment related toxicity associated with escalating neoadjuvant RT dose. A concern with the use of higher neoadjuvant RT doses is greater patient toxicity during radiation as well as greater perioperative morbidity. The NCDB does not have information regarding the morbidity experienced by patients during radiation or in the immediate postoperative period per se, but does contain information regarding 30 and 90 -day post operative mortality. This is a reasonable surrogate for operative morbidity, and the investigators were able to demonstrate worse 90 -day post operative mortality in the hRT cohort, suggesting that this level of RT dose may cause excessive toxicity. 
This study also had some weaknesses that may limit the generalizability of its findings. One, the authors only used neoadjuvant RT as a criterion for entry, and not neoadjuvant CRT. The standard neoadjuvant treatment based on the CROSS trial is neoadjuvant CRT (2), as this is the treatment arm that was demonstrated to have superior OS when compared to surgery alone. While it is true that about $90 \%$ of patients in each dose cohort did receive chemotherapy, there were significant differences in patients receiving multi- agent chemotherapy. It is possible that differences in pCR rate in the different dose cohorts would not have been observed if the authors had restricted the analysis to patients receiving cRT. Additionally, there were significant differences in the interval from radiation to operation in the different dose cohorts. For example, $43.6 \%$ of patients receiving high-dose RT had surgery $>105$ days after radiation, whereas only $24.1 \%$ of patients receiving standard-dose RT had surgery $>105$ days after radiation. It is possible that the increased interval from surgery to RT in the high dose RT arm allowed these patients to experience a higher pCR rate. Finally, since this was a retrospective review, an intent- to- treat analysis could not have been done, and the reasons for the specific dose selected could not be explained.

The study by Semenkovich et al. (10) is a good effort to further explore the dose response effect observed when comparing different neoadjuvant RT doses. As expected, higher neoadjuvant RT doses were associated with higher pCR rates. Interestingly, the hRT dose cohort was also observed to have the worst 90-day post operative mortality, suggesting that this dose range may lead to an unacceptable level of perioperative morbidity. Unfortunately, the study did not control for chemotherapy use, nor did it attempt to standardize the time from radiation to surgery, both of which could have skewed the results. Regardless, the conclusion neoadjuvant RT doses from 40-50.4 Gy are optimal be used in the neoadjuvant setting to maximize the benefit of RT while minimizing the harm is a reasonable one to make based on the data presented herein. Further work should be performed to determine if this remains true in the setting of concurrent chemotherapy, and if a dose of 40-41.4 Gy is sufficient, or whether escalation to $50.4 \mathrm{~Gy}$ is required in order to obtain the beneficial effects of neoadjuvant treatment.

\section{Acknowledgments}

None.

\section{Footnote}

Conflicts of Interest: The authors have no conflicts of interest to declare.

Ethical Statement: The authors are accountable for all aspects of the work in ensuring that questions related to the accuracy or integrity of any part of the work are appropriately investigated and resolved.

\section{References}

1. National Comprehensive Cancer Network Guidelines Version 2. 2019. Esophageal and Esophagogastric Junction Cancers. Accessed November 2019. Available online: http://nccn.rg/profesionals/physician_gls/pdf/ prostate.pdf

2. van Hagen P, Hulshof MC, van Lanschot JJ, et al. Preoperative chemoradiotherapy for esophageal or junctional cancer. N Engl J Med 2012;366:2074-84.

3. Tepper J, Krasna MJ, Niedzwiecki D, et al. Phase III trial of trimodality therapy with cisplatin, fluorouracil, radiotherapy and surgery compared with surgery alone for esophageal cancer: CALGB 9781. J Clin Oncol 2008;26:1086-92.

4. Walsh TN, Noonan N, Hollywood D, et al. A comparison of multimodal therapy and surgery for esophageal adenocarcinoma. N Engl J Med 1996;335:462-7.

5. Urba SG, Orringer MB, Turrisi A, et al. Randomized trial of preoperative chemoradiation versus surgery alone in patients with locoregional esophageal carcinoma. J Clin Oncol 2001;19:305-13.

6. Burmeister BH, Smithers BM, Gebski V, et al. Surgery alone versus chemoradiotherapy followed by surgery for resectable cancer of the oesophagus: a randomized controlled phase III trial. Lancel Oncol 2015;6:659-68.

7. Swisher SG, Marks J, Rice D, et al. Salvage esophagectomy for persistent or recurrent disease after definitive chemoradiation. Ann Cardiothorac Surg 2017;6:144-51.

8. Minsky BD, Neuberg D, Kelsen DP, et al. Final report of intergroup trial 0122 (ECOG PE-289, RTOG 9012): phase II trial of neoadjuvant chemotherapy plus concurrent chemotherapy and high-dose radiation for squamous cell carcinoma of the esophagus. Int J Radiat Oncol Biol Phys 1999;43:517-23.

9. Minsky BD, Pajak TF, Ginsberg RJ, et al. INT 0123 
(Radiation therapy oncology group 94-05) phase III trial of combined- modality therapy for esophageal cancer: high- dose versus standard-dose radiation therapy. J Clin Oncol 2002;20:1167-74.

Cite this article as: Haque W, Butler EB, Teh BS. Neoadjuvant radiation therapy for esophageal cancer: is a higher dose better? J Thorac Dis 2019;11(12):5631-5634. doi: 10.21037/jtd.2019.11.77
10. Semenkovich TR, Samson PP, Hudson JL, et al. Induction radiation therapy for esophageal cancer: does dose affect outcomes? Ann Thorac Surg 2019;107:903-11. 